\title{
Simulation of Natural Convective Boundary Layer Flow of a Nanofluid Past a Convectively Heated Inclined Plate in the Presence of Magnetic Field
}

\author{
Mania Goyal $^{1}$ - Rama Bhargava ${ }^{2}$
}

Published online: 24 February 2018

(C) The Author(s) 2018. This article is an open access publication

\begin{abstract}
This paper deals with the numerical simulation of transient magnetohydrodynamics natural convective boundary layer flow of a nanofluid over an inclined plate. In the modeling of nanofluids, dynamic effects including the Brownian motion and thermophoresis are taken into account. Numerical solutions have been computed via the Galerkin-finite element method. The effects of angle of inclination, buoyancy-ratio parameter, Brownian motion, thermophoresis and magnetic field are taken into account and controlled by nondimensional parameters. To compute the rate of convergence and error of the computed numerical solution, the double mesh principle is used. Similarity solutions are calculated and presented graphically for non-dimensional velocity, temperature, local rate of heat and mass transfer with pertinent parameters. The modified Nusselt number decreases with increasing inclination angle, buoyancy-ratio parameter, Brownian motion and thermophoresis parameter, whereas it increases with increasing Prandtl number. Validation of the results is achieved with earlier results for forced convective flow and non-magnetic studies. Such problems have several applications in engineering and petroleum industries such as electroplating, chemical processing of heavy metals and solar water heaters. External magnetic fields play an important role in electrical power generation, inclination/acceleration sensors, fine-tuning of the final materials to industrial specification because of their controlling behaviour on the flow characteristics of nanofluids.
\end{abstract}

Keywords Nanofluid · MHD - Inclined plate - Similarity solution · Convective boundary condition · Finite element method · Double mesh principle

Mania Goyal

goyalmania87@gmail.com

Rama Bhargava

rbharfma@iitr.ernet.in

1 Materials and Engineering Research Institute, Sheffield Hallam University, Sheffield S11WB, UK

2 Department of Mathematics, Indian Institute of Technology Roorkee, Roorkee 247667, India 


\section{List of symbols}

\section{Roman}

$B_{o} \quad$ Uniform magnetic field strength

$D_{B} \quad$ Brownian diffusion coefficient

$D_{T} \quad$ Thermophoresis diffusion coefficient

$E_{N} \quad$ Error

$f \quad$ Dimensionless stream function

$g_{e} \quad$ Acceleration due to gravity

$h \quad$ Dimensionless velocity function

$h_{e} \quad$ Step size

$h_{f} \quad$ Heat transfer coefficient

$k \quad$ Thermal conductivity

Ln Nanofluid Lewis number

$M \quad$ Dimensionless magnetic parameter

$\mathrm{Nb} \quad$ Brownian motion parameter

Nc Convective heating parameter

$\mathrm{Nr} \quad$ Buoyancy-ratio parameter

$N t \quad$ Thermophoresis parameter

$N u_{x} \quad$ Local Nusselt number

Nur Reduced Nusselt number

$\mathrm{Pr} \quad$ Prandtl number

$R a_{x} \quad$ Local Rayleigh number

$r^{N} \quad$ Rate of convergence

$S h_{x, n}$ Local nanoparticle Sherwood number

Shrn Reduced nanoparticle Sherwood number

$T \quad$ Fluid temperature

$T_{f} \quad$ Hot fluid temperature

$T_{w} \quad$ Fluid temperature at the wall

$T_{\infty} \quad$ Ambient temperature

$u, v \quad$ Velocity components along $x$ and $y$-directions

\section{Greek symbols}

$\alpha_{m} \quad$ Thermal diffusivity

$\beta \quad$ Thermal expansion coefficient

$\delta \quad$ Acute angle of the plate to the vertical

$\mu \quad$ Viscosity of the nanofluid

$v \quad$ Kinematic viscosity of the fluid

$\phi \quad$ Dimensionless nanoparticle volume fraction

$\hat{\phi} \quad$ Nanoparticle volume fraction

$\hat{\phi}_{w} \quad$ Nanoparticle volume fraction at the wall

$\hat{\phi}_{\infty} \quad$ Ambient nanoparticle volume fraction

$\psi \quad$ Stream function

$\rho_{f} \quad$ Density of the nanofluid

$\sigma_{n f} \quad$ Electrical conductivity of the nanofluid 
$\tau \quad$ Ratio between the effective heat capacity of the nanoparticle material and heat capacity of the fluid, defined by $\left(\rho c_{p}\right)_{p} /\left(\rho c_{p}\right)_{f}$

$\theta \quad$ Dimensionless temperature

\title{
Subscripts
}

\author{
$f \quad$ Base fluid \\ $n f \quad$ Nanofluid \\ $p \quad$ Nanoparticle \\ $w, \infty$ Condition at the surface and in the free stream, respectively
}

\section{Introduction}

Nanoparticles provide a connection between molecular structure and bulk materials. When nanoparticles strategically deployed in the base fluids, the ensuing nanofluids have been verified to achieve remarkable enhancement in the properties of thermal conductivity, as introduced by Choi [8]. This has made nanofluids attractive in various areas of recent technology incorporating heat exchangers [15], aerospace cooling systems [20], and energy systems [17]. The two most common approaches to investigate the phenomena of heat and mass transfer characteristics are either the Tiwari and Das model [26] (which only requires momentum and energy equations and incorporates nanoparticle effects via a volume fraction parameter only) and the Buongiorno non-homogeneous model [6] (which introduces a separate equation for the nanoparticle concentration). Several researchers worked on these models including Hatami et al. [14], Goyal and Bhargava [12], Hamad et al. [13].

The natural convection exerts a significant influence on the heat and mass transfer analysis in the problems of nanofluids. In most of the fluid flow processes, transport phenomena occur due to the combined effect of heat and mass transfer. This is because of buoyancy effects arising from density variation, which is due to variation in temperature and/or concentration of particles. The classical problem, which involves natural convective flow of a regular fluid over a vertical plate, was first investigated theoretically by Pohlhausen [22]. Thereafter, Bejan [5] incorporated the effect of Prandtl number on boundary layers in natural convective fluid flow problems. An extension of the classical problem [22] to incorporate the effect of heat and mass transfer was investigated by Khair and Bejan [16]. Later, Aziz and Khan [4] numerically investigated the free convective boundary layer flow of a nanofluid over a vertical plate. Their analysis showed that the flow pattern, heat and mass transfer analysis strongly influenced by the pertinent parameters.

Lately, the problems of free convection fluid flow over a plate for different values of inclination angle were frequently encountered in engineering devices such as solar water heaters and inclination/acceleration sensors. Most of the researchers $[1,3,7]$ observed that fluid flow through the medium was favoured in case of an inclined plate as inclination to the vertical reduces the drag force. A generalized formulation was explained by Ali et al. [2] for the combined effect of chemical reaction and radiation on MHD free convective flow of viscous fluid over an inclined plate. They found that the flow features not only depend on the magnitude of inclination but also on the distance from the leading edge. Later, Narahari et al. [19] has studied the effect of free convective flow of a nanofluid over an isothermal inclined plate and observed that the thickness of the momentum boundary layer decreases with an increase in angle of inclination whereas the temperature and nanoparticle volume fraction increase with increasing inclination angle. 
The study of flow analysis and heat transfer under the influence of an applied magnetic field is considered a significant research topic due to its numerous scientific, industrial and biological applications such as crystal growth, cooling of metallic plates, production of magnetorheostatic materials known as smart fluids, metal casting and liquid metal cooling blankets for fusion reactors. The rate of heat transfer can be controlled by MHD flow in electrically conducting fluid and hence desired cooling effect can be achieved. The different types of thermal boundary conditions were used by Sathiyamoorthy and Chamkha [23] to study steady state, laminar, 2D natural convective flow in the presence of an inclined magnetic field in a square enclosure filled with liquid Gallium. Recently, Goyal and Bhargava [9] numerically investigated the MHD viscoelastic nanofluid flow past a stretching sheet with heat source/sink and partial slip. It was observed from the study that modified Nusselt number is directly proportional to Brownian motion and thermophoretic parameters and indirectly proportional to all other parameters.

As, the study of a convectively heated inclined plate plays an important role in many processes such as manufacturing of tetrapacks, glass fibres, plastic and rubber sheets, solidification of casting. An efficient manufacturing of such materials incorporates various physical phenomena including the implementation of magnetohydrodynamics (MHD), thermal and mass diffusion effects at nanoscale level. To improve the interpretation of the inter-disciplinary transport phenomena in such type of systems, a robust approach is provided with the help of mathematical model. Hence, motivated by this, the present study focused to develop a mathematical model for natural convective boundary layer flow of a nanofluid past a convectively heated inclined plate in the presence of Magnetic field.

The Buongiorno nanofluid model approach [6] is used which emphasizes the Brownian motion and thermophoresis effects. This approach also introduces a separate equation for nano-particle species diffusion. By using the suitable similarity transformation for velocity, temperature and nanoparticle concentration, the equations governing for flow, heat and mass transfer were transformed to a set of ordinary differential equations. The resulting equations subjected to the boundary conditions were solved numerically using conventional finite element method (FEM). The numerical investigation is carried out for different thermophysical parameters, namely: the magnetic parameter, buoyancy-ratio parameter, convective heat parameter, Prandtl number, nanofluid Lewis number, Brownian motion parameter, and thermophoresis parameter. The obtained results are validated by comparing with work of other authors that has reported in literature. The rates of heat and nano-mass transfer were computed and were shown in both tabular and graphical formats.

\section{Problem Formulation}

\section{Governing Equations and Boundary Conditions}

The flow of fluid was assumed to be steady, incompressible, two-dimensional and laminar with constant physical properties. The semi-infinite plate was inclined at an acute angle $\delta$ to the vertical axis. With $x$-axis measured along the plate, a magnetic field of uniform strength $B_{o}$ was applied in the $y$-direction (normal to the flow direction). The gravitational acceleration $g_{e}$ was acting downward. In addition, the buoyancy effects were included in momentum transfer with the usual Boussinesq approximation. It was also assumed that the 
Fig. 1 Physical model and co-ordinate system

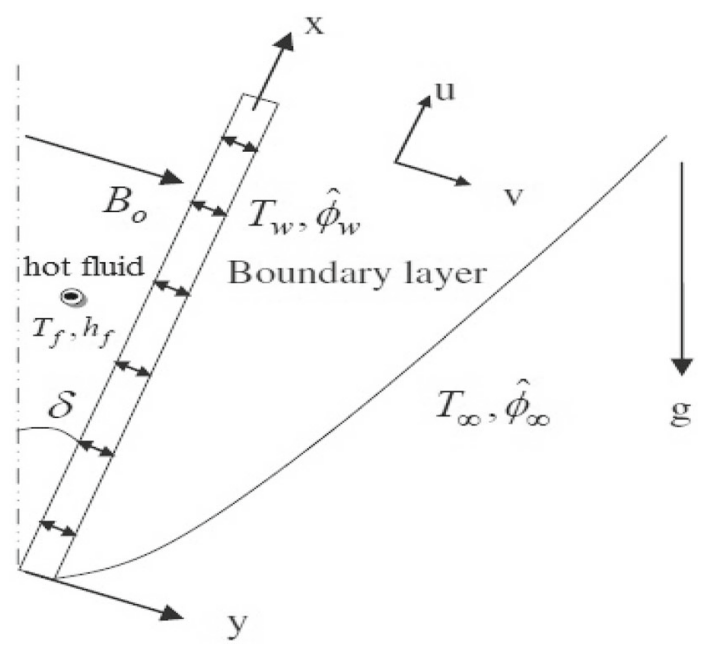

lower side of the plate was heated by convection through a hot fluid at temperature $T_{f}$ and with coefficient of heat transfer $h_{f}$. It was assumed that both the nanoparticles and the base fluid are in thermal equilibrium. In the vicinity of the plate, three different types of boundary layers (momentum, thermal and nanoparticle volume fraction) were formed. The physical configuration of the problem is shown in Fig. 1.

Upon incorporating the main assumptions into the conservation equations for mass, momentum, thermal energy and nanoparticle species, the dimensional set of governing equations is written as:

$$
\begin{aligned}
\frac{\partial u}{\partial x}+\frac{\partial v}{\partial y}= & 0 \\
\rho_{f}\left(u \frac{\partial u}{\partial x}+v \frac{\partial u}{\partial y}\right)= & \mu \frac{\partial^{2} u}{\partial y^{2}}-\sigma_{n f} B_{o}^{2} u+\left[\left(1-\hat{\phi}_{\infty}\right) \rho_{f_{\infty}} \beta g_{e}\left(T-T_{\infty}\right)\right. \\
& \left.\left.-\left(\rho_{p}-\rho_{f_{\infty}}\right) g_{e}\left(\hat{\phi}-\hat{\phi}_{\infty}\right)\right)\right] \cos \delta \\
u \frac{\partial T}{\partial x}+v \frac{\partial T}{\partial y}= & \alpha_{m} \frac{\partial^{2} T}{\partial y^{2}}+\tau\left[D_{B} \frac{\partial \hat{\phi}}{\partial y} \frac{\partial T}{\partial y}+\frac{D_{T}}{T_{\infty}}\left(\frac{\partial T}{\partial y}\right)^{2}\right] \\
u \frac{\partial \hat{\phi}}{\partial x}+v \frac{\partial \hat{\phi}}{\partial y}= & D_{B} \frac{\partial^{2} \hat{\phi}}{\partial y^{2}}+\frac{D_{T}}{T_{\infty}} \frac{\partial^{2} T}{\partial y^{2}}
\end{aligned}
$$

where $u$ and $v$ are the velocity components parallel and perpendicular to the plate, respectively, $B_{O}$ is uniform magnetic field strength, $\hat{\phi}$ is the local solid volume fraction of the nanoparticles, $\beta$ is volumetric thermal expansion coefficient of the base fluid, $D_{B}$ is the Brownian diffusion coefficient, $D_{T}$ is the thermophoretic diffusion coefficient, and $T$ is the local temperature.

Continuity, momentum, thermal energy, and nanoparticle species equations for nanofluid are represented by Eqs. (1)-(4), respectively. The terms (from left to right) in the right side of Eq. (2) represent the stress component due to viscosity, the convective acceleration and the force due to the magnetic field. The first and second terms in the square bracket in (2) represent the positive (upward) buoyancy term due to the thermal expansion of the base fluid and the negative (downward) buoyancy term due to the variation in densities of the nanoparticles and the base fluid, respectively. The terms in the left hand side of Eq. (3) are 
the convection terms due to temperature. On the other hand, the terms on right side (left to right) represent the heat enthalpy, diffusion of thermal energy due to Brownian diffusion and thermophoretic effect. A similar interpretation could be given to the terms on the right hand side of Eq. (4).

The boundary conditions may be written as

$$
\begin{aligned}
& u=0, v=0, \hat{\phi}=\hat{\phi}_{w}, \quad-k \frac{\partial T}{\partial y}=h_{f}\left(T_{f}-T\right) \text { at } y=0 \\
& u=0, v=0, \hat{\phi}=\hat{\phi}_{\infty}, \quad T=T_{\infty} \text { as } y \rightarrow \infty
\end{aligned}
$$

\section{Similarity Transformations}

By introducing the stream function $\psi$, with $u=\partial \psi / \partial y$ and $v=-\partial \psi / \partial x$, the system of Eqs. (1)-(4) reduces to

$$
\begin{aligned}
& 0=\mu \frac{\partial^{3} \psi}{\partial y^{3}}-\rho_{f}\left(\frac{\partial \psi}{\partial y} \frac{\partial^{2} \psi}{\partial x \partial y}-\frac{\partial \psi}{\partial x} \frac{\partial^{2} \psi}{\partial y^{2}}\right)-\sigma_{n f} B_{o}^{2} \frac{\partial \psi}{\partial y}+\left[\left(1-\hat{\phi}_{\infty}\right) \rho_{f_{\infty}} \beta g_{e}\right. \\
& \left.\left(T-T_{\infty}\right)-\left(\rho_{p}-\rho_{f_{\infty}} g_{e}\left(\hat{\phi}-\hat{\phi}_{\infty}\right)\right)\right] \cos \delta \\
& \frac{\partial \psi}{\partial y} \frac{\partial T}{\partial x}-\frac{\partial \psi}{\partial x} \frac{\partial T}{\partial y}=\alpha_{m} \frac{\partial^{2} T}{\partial y^{2}}+\tau\left[D_{B} \frac{\partial \hat{\phi}}{\partial y} \frac{\partial T}{\partial y}+\frac{D_{T}}{T_{\infty}}\left(\frac{\partial T}{\partial y}\right)^{2}\right] \\
& \frac{\partial \psi}{\partial y} \frac{\partial \hat{\phi}}{\partial x}-\frac{\partial \psi}{\partial x} \frac{\partial \hat{\phi}}{\partial y}=D_{B} \frac{\partial^{2} \hat{\phi}}{\partial y^{2}}+\frac{D_{T}}{T_{\infty}} \frac{\partial^{2} T}{\partial y^{2}}
\end{aligned}
$$

The following similarity transformation are used in order to non-dimensionlize the system of differential Eqs. (1)-(4):

$$
\eta=\frac{y}{x} R a_{x}^{1 / 4}, \psi=\alpha_{m} R a_{x}^{1 / 4} f(\eta), \theta(\eta)=\frac{T-T_{\infty}}{T_{f}-T_{\infty}}, \phi(\eta)=\frac{\hat{\phi}-\hat{\phi}_{\infty}}{\hat{\phi}_{w}-\hat{\phi}_{\infty}}
$$

with the local Rayleigh number is defined as

$$
R a_{x}=\frac{\left(1-\hat{\phi}_{\infty}\right) \beta g_{e}\left(T_{f}-T_{\infty}\right) x^{3}}{\nu \alpha_{m}}
$$

Now

$$
\begin{aligned}
\frac{\partial \eta}{\partial x} & =-\frac{1}{4} \frac{y}{x^{2}} R a_{x}^{1 / 4}, \\
\frac{\partial \eta}{\partial y} & =\frac{1}{x} R a_{x}^{1 / 4}, \\
u & =\frac{\partial \psi}{\partial y}=\alpha_{m} R a_{x}^{1 / 4} \frac{\partial f}{\partial \eta} \frac{\partial \eta}{\partial y}=\frac{\alpha_{m}}{x} R a_{x}^{1 / 2} f^{\prime}(\eta), \\
v & =-\frac{\partial \psi}{\partial x}=-\alpha_{m}\left(R a_{x}^{1 / 4} \frac{\partial f}{\partial \eta} \frac{\partial \eta}{\partial x}+\frac{1}{4} R a_{x}^{-3 / 4} \frac{3 R a_{x}}{x}\right) \\
& =\frac{y}{4 x^{2}} \alpha_{m} R a_{x}^{1 / 2} f^{\prime}(\eta)-\frac{3}{4 x} \alpha_{m} R a_{x}^{1 / 4} f(\eta), \\
\frac{\partial u}{\partial x} & =\frac{1}{2} \frac{\alpha_{m}}{x^{2}} R a_{x}^{1 / 2} f^{\prime}(\eta)-\frac{y}{4 x^{2}} \frac{\alpha_{m}}{x} R a_{x}^{3 / 4} f^{\prime \prime}(\eta), \\
\frac{\partial u}{\partial y} & =\frac{\alpha_{m}}{x} R a_{x}^{3 / 4} \frac{\partial f^{\prime}}{\partial \eta} \frac{\partial \eta}{\partial y}=\frac{\alpha_{m}}{x^{2}} R a_{x}^{3 / 4} f^{\prime \prime}(\eta),
\end{aligned}
$$




$$
\frac{\partial^{2} u}{\partial y^{2}}=\frac{\alpha_{m}}{x^{2}} R a_{x}^{3 / 4} \frac{\partial f^{\prime \prime}}{\partial \eta} \frac{\partial \eta}{\partial y}=\frac{\alpha_{m}}{x^{3}} R a_{x} f^{\prime \prime \prime}(\eta) .
$$

Substituting (12), (13) in Eq. (1), we have

$$
\begin{aligned}
\frac{\alpha_{m}}{x} & R a_{x}^{1 / 2} f^{\prime}\left(\frac{\alpha_{m}}{2 x^{2}} R a_{x}^{1 / 2} f^{\prime}-\frac{\alpha_{m}}{4 x} \frac{y}{x^{2}} R a_{x}^{3 / 4} f^{\prime \prime}\right) \\
& -\left(-\frac{y}{4 x^{2}} \alpha_{m} R a_{x}^{1 / 2} f^{\prime}+\frac{3}{4} \frac{\alpha_{m}}{x} R a_{x}^{1 / 4} f\right) \frac{\alpha_{m}}{x^{2}} R a_{x}^{3 / 4} f^{\prime \prime} \\
= & \frac{\mu}{\rho_{f}} \frac{\alpha_{m}}{x^{3}} R a_{x} f^{\prime \prime \prime}-\frac{\sigma_{n f} B_{o}^{2}}{\rho_{f}} \frac{\alpha_{m}}{x} R a_{x}^{1 / 2} f^{\prime} \\
& \left.+\frac{1}{\rho_{f}}\left(\left(1-\hat{\phi}_{\infty}\right) \rho_{f_{\infty}} \beta g_{e}\left(T_{f}-T_{\infty}\right) \theta-\left(\rho_{f}-\rho_{f_{\infty}}\right) g_{e}\left(\hat{\phi}_{w}-\hat{\phi}_{\infty}\right) \phi\right)\right) \cos \delta
\end{aligned}
$$

With simple calculations, the above equation can be written as:

$$
\begin{aligned}
& \frac{\alpha_{m}}{2 x^{3}} R a_{x} f^{\prime 2}-\frac{3 \alpha_{m}^{2}}{x^{3}} R a_{x} f f^{\prime \prime}=\frac{\mu}{\rho_{f}} \frac{\alpha_{m}}{x^{3}} R a_{x} f^{\prime \prime \prime}+\frac{\left(1-\hat{\phi}_{\infty}\right) \rho_{f_{\infty}} \beta g_{e}\left(T_{f}-T_{\infty}\right)}{\rho_{f}} \\
& \left(\theta-\frac{\left(\rho_{f}-\rho_{f_{\infty}}\right) g\left(\rho_{f}-\rho_{f_{\infty}}\right)}{\left(1-\hat{\phi}_{\infty}\right) \rho_{f_{\infty}} \beta g_{e}\left(T_{f}-T_{\infty}\right)}\right) \cos \delta-\frac{\sigma_{n f} B_{o}^{2}}{\rho_{f}} \frac{\alpha_{m}}{x} R a_{x}^{1 / 2} f^{\prime} \\
& \Rightarrow \frac{\alpha_{m}^{2}}{4 x^{3}}\left(2 f^{\prime 2}-3 f f^{\prime \prime}\right)=\frac{\mu}{\rho_{f}} \frac{\alpha_{m}}{x^{3}} R a_{x} f^{\prime \prime \prime}+\frac{\left(1-\hat{\phi}_{\infty}\right) \rho_{f_{\infty}} \beta g\left(T_{f}-T_{\infty}\right)}{\rho_{f}} \\
& (\theta-N r \phi) \cos \delta-\frac{\sigma B_{o}^{2}}{\rho_{f}} \frac{\alpha_{m}}{x} R a_{x}^{1 / 2} f^{\prime},
\end{aligned}
$$

which implies that

$$
\begin{aligned}
f^{\prime \prime \prime} & +\frac{\alpha_{m}}{4 \mu} \rho_{f}\left(3 f f^{\prime \prime}-2 f^{\prime 2}\right)+(\theta-N r \phi) \cos \delta-\frac{\sigma B_{o}^{2} x^{3}}{\mu R a_{x}^{1 / 2}} f^{\prime}=0 \\
\Rightarrow f^{\prime \prime \prime}+\frac{1}{4 P r}\left(3 f f^{\prime \prime}-2 f^{\prime 2}\right)+(\theta-N r \phi) \cos \delta-M f^{\prime} & =0
\end{aligned}
$$

where prime denote differentiation with respect to $\eta$ and the parameters $\operatorname{Pr}$ (Prandtl number), $N r$ (Buoyancy-ratio parameter) and $M$ (magnetic parameter) appearing in Eq. (15) are defined as:

$$
P r=\frac{\mu}{\alpha_{m}}, \quad N r=\frac{\left(\rho_{p}-\rho_{f}\right)\left(\hat{\phi}_{w}-\hat{\phi}_{\infty}\right)}{\rho_{f} \beta\left(1-\hat{\phi}_{w}\right)\left(T_{f}-T_{\infty}\right)}, \quad M=\frac{\sigma_{n f} B_{o}^{2} x^{3}}{\mu R a_{x}^{1 / 2}}
$$

In order to non-dimensionlize the energy equation (3), the following terms are computed as:

$$
\begin{aligned}
\frac{\partial T}{\partial y} & =\left(T_{f}-T_{\infty}\right) \frac{\partial \theta}{\partial \eta} \frac{\partial \eta}{\partial y}=\frac{\left(T_{f}-T_{\infty}\right)}{x} R a_{x}^{1 / 4} \theta^{\prime}(\eta), \\
\frac{\partial^{2} T}{\partial y^{2}} & =\frac{\partial}{\partial y}\left(\frac{\left(T_{f}-T_{\infty}\right)}{x} R a_{x}^{1 / 4} \theta^{\prime}(\eta)\right)=\frac{\left(T_{f}-T_{\infty}\right)}{x} R a_{x}^{1 / 4} \frac{\partial^{2} \theta}{\partial \eta^{2}} \frac{\partial \eta}{\partial y} \\
& =\frac{\left(T_{f}-T_{\infty}\right)}{x^{2}} R a_{x}^{1 / 2} \theta^{\prime \prime}(\eta), \\
\frac{\partial T}{\partial x} & =\left(T_{f}-T_{\infty}\right) \frac{\partial \theta}{\partial \eta} \frac{\partial \eta}{\partial x}=-\frac{y}{4 x^{2}}\left(T_{f}-T_{\infty}\right) R a_{x}^{1 / 4} \theta^{\prime}(\eta),
\end{aligned}
$$


and

$$
\begin{aligned}
\frac{\partial \hat{\phi}}{\partial y} & =\left(\hat{\phi}_{w}-\hat{\phi}_{\infty}\right) \frac{\partial \phi}{\partial \eta} \frac{\partial \eta}{\partial y}=\frac{\left(\hat{\phi}_{w}-\hat{\phi}_{\infty}\right)}{x} R a_{x}^{1 / 4} \phi^{\prime}(\eta), \\
\frac{\partial^{2} \hat{\phi}}{\partial y^{2}} & =\frac{\partial}{\partial y}\left(\frac{\left(\hat{\phi}_{w}-\hat{\phi}_{\infty}\right)}{x} R a_{x}^{1 / 4} \phi^{\prime}(\eta)\right)=\frac{\left(\hat{\phi}_{w}-\hat{\phi}_{\infty}\right)}{x} R a_{x}^{1 / 4} \frac{\partial^{2} \phi}{\partial \eta^{2}} \frac{\partial \eta}{\partial y} \\
& =\frac{\left(\hat{\phi}_{w}-\hat{\phi}_{\infty}\right)}{x^{2}} R a_{x}^{1 / 2} \phi^{\prime \prime}(\eta), \\
\frac{\partial \hat{\phi}}{\partial x} & =\left(\hat{\phi}_{w}-\hat{\phi}_{\infty}\right) \frac{\partial \phi}{\partial \eta} \frac{\partial \eta}{\partial x}=-\frac{y}{4 x^{2}}\left(\hat{\phi}_{w}-\hat{\phi}_{\infty}\right) R a_{x}^{1 / 4} \phi^{\prime}(\eta) .
\end{aligned}
$$

Now substituting these values from Eqs. (12), (13), (18) and (19) in (3), we obtain

$$
\begin{aligned}
- & \frac{\alpha_{m}}{x} R a_{x}^{1 / 2} f^{\prime}(\eta) \frac{y}{4 x^{2}}\left(T_{f}-T_{\infty}\right) R a_{x}^{1 / 4} \theta^{\prime}(\eta) \\
& -\left(-\frac{y}{4 x^{2}} \alpha_{m} R a_{x}^{1 / 2} f^{\prime}(\eta)+\frac{3}{4 x} \alpha_{m} f(\eta) R a_{x}^{1 / 4}\right) \\
& \times \frac{\left(T_{f}-T_{\infty}\right)}{x} R a_{x}^{1 / 4} \theta^{\prime}(\eta)=\alpha_{m} \frac{\left(T_{f}-T_{\infty}\right)}{x^{2}} R a_{x}^{1 / 2} \theta^{\prime \prime}(\eta) \\
& +\tau\left(D_{B} \frac{\left(\hat{\phi}_{w}-\hat{\phi}_{\infty}\right)}{x} \frac{\left(T_{f}-T_{\infty}\right)}{x} R a_{x}^{1 / 2} \theta^{\prime} \phi^{\prime}+\frac{D_{T}}{T_{\infty}} \frac{\left(T_{f}-T_{\infty}\right)^{2}}{x^{2}} \theta^{\prime 2}\right), \\
\Rightarrow & -\frac{\alpha_{m}}{4} \frac{y}{x^{3}}\left(T_{f}-T_{\infty}\right) R a_{x}^{3 / 4} f^{\prime} \theta^{\prime}+\frac{1}{4} \frac{\alpha_{m}}{x} \frac{y}{x^{2}}\left(T_{f}-T_{\infty}\right) R a_{x}^{3 / 4} f^{\prime} \theta^{\prime} \\
& -\frac{3}{4 x^{2}} \alpha_{m}\left(T_{f}-T_{\infty}\right) R a_{x}^{1 / 2} f \theta^{\prime}=\frac{\alpha_{m}}{x^{2}}\left(T_{f}-T_{\infty}\right) R a_{x}^{1 / 2} \theta^{\prime \prime} \\
& +\left(\frac{D_{B}}{x^{2}}\left(\hat{\phi}_{w}-\hat{\phi}_{\infty}\right)\left(T_{f}-T_{\infty}\right) R a_{x}^{1 / 2} \theta^{\prime} \phi^{\prime}+\frac{D_{T}}{T_{\infty}} \frac{\left(T_{f}-T_{\infty}\right)^{2}}{x^{2}} \theta^{\prime 2}\right)
\end{aligned}
$$

Leading to:

$$
\begin{aligned}
& -\frac{3}{4} \alpha_{m} f \theta^{\prime}=\alpha_{m} \theta^{\prime \prime}+\tau\left(D_{B}\left(\hat{\phi}_{w}-\hat{\phi}_{\infty}\right) \theta^{\prime} \phi^{\prime}+\frac{D_{T}}{T_{\infty}}\left(T_{f}-T_{\infty}\right) \theta^{\prime 2}\right), \\
& \Rightarrow \theta^{\prime \prime}+\frac{3}{4} f \theta^{\prime}+\frac{\tau}{\alpha_{m}} D_{B}\left(\hat{\phi}_{w}-\hat{\phi}_{\infty}\right) \theta^{\prime} \phi^{\prime}+\tau \frac{D_{T}}{T_{\infty}} \frac{\left(T_{f}-T_{\infty}\right)}{\alpha_{m}} \theta^{\prime 2}=0, \\
& \Rightarrow \theta^{\prime \prime}+\frac{3}{4} f \theta^{\prime}+N b \theta^{\prime} \phi^{\prime}+N t \theta^{\prime 2}=0
\end{aligned}
$$

where $\mathrm{Nb}$ (Brownian motion parameter) and $\mathrm{Nt}$ (thermophoresis parameter) appearing in equation (24) are defined as:

$$
N b=\frac{\tau D_{B}\left(\hat{\phi}_{w}-\hat{\phi}_{\infty}\right)}{\alpha_{m}}, \quad N t=\frac{\tau D_{T}\left(T_{f}-T_{\infty}\right)}{T_{\infty} \alpha_{m}}
$$

Now substituting the values from Eqs. (12), (13), (18) and (19) in the nanoparticle concentration equation (3), we get

$$
\begin{aligned}
& -\frac{\alpha_{m}}{x} R a_{x}^{1 / 2} f^{\prime} \frac{y}{4 x^{2}} R a_{x}^{1 / 4} \phi^{\prime}-\left(-\frac{y}{4 x^{2}} \alpha_{m} R a_{x}^{1 / 2} f^{\prime}+\frac{3}{4 x} \alpha_{m} R a_{x}^{1 / 4} f\right) \\
& \frac{\left(\hat{\phi}_{w}-\hat{\phi}_{\infty}\right)}{x} R a_{x}^{1 / 4} \phi^{\prime}=\frac{D_{B}\left(\hat{\phi}_{w}-\hat{\phi}_{\infty}\right)}{x^{2}} R a_{x}^{1 / 2} \phi^{\prime \prime}+\frac{D_{T}}{T_{\infty}} \frac{\left(T_{f}-T_{\infty}\right)}{x^{2}} R a_{x}^{1 / 2} \theta^{\prime \prime}
\end{aligned}
$$




$$
\begin{aligned}
- & \frac{3}{4 x^{2}} \alpha_{m} R a_{x}^{1 / 2}\left(\hat{\phi}_{w}-\hat{\phi}_{\infty}\right) f \phi^{\prime} \\
= & \frac{D_{B}}{x^{2}}\left(\hat{\phi}_{w}-\hat{\phi}_{\infty}\right) R a_{x}^{1 / 2} \phi^{\prime \prime}+\frac{D_{T}}{T_{\infty}} \frac{\left(T_{f}-T_{\infty}\right)}{x^{2}} R a_{x}^{1 / 2} \theta^{\prime \prime}
\end{aligned}
$$

or

$$
\begin{aligned}
& -\frac{3}{4} \alpha_{m} f \phi^{\prime}=D_{B} \phi^{\prime \prime}+\frac{D_{T}}{T_{\mid} i n f t y} \frac{\left(T_{f}-T_{\infty}\right)}{\left(\hat{\phi}_{w}-\hat{\phi}_{\infty}\right)} \theta^{\prime \prime}, \\
& \Rightarrow \phi^{\prime \prime}+\frac{1}{D_{B}} \frac{D_{T}}{T_{\mid} \text {infty }} \frac{\left(T_{f}-T_{\infty}\right)}{\left(\hat{\phi}_{w}-\hat{\phi}_{\infty}\right)} \theta^{\prime \prime}, \\
& \Rightarrow \phi^{\prime \prime}+\frac{N t}{N b} \theta^{\prime \prime}+\frac{3}{4} \operatorname{Lnf\phi ^{\prime }}=0
\end{aligned}
$$

where $\operatorname{Ln}$ (nanofluid Lewis number) is defined by $\operatorname{Ln}=\frac{\alpha_{m}}{D_{B}}$.

Finally, the following system of non-dimensionlize is obtained as

$$
\begin{aligned}
& f^{\prime \prime \prime}+\frac{1}{4 P r}\left(3 f f^{\prime \prime}-2 f^{\prime 2}\right)+(\theta-N r \phi) \cos \delta-M f^{\prime}=0 \\
& \theta^{\prime \prime}+\frac{3}{4} f \theta^{\prime}+N b \theta^{\prime} \phi^{\prime}+N t \theta^{\prime 2}=0 \\
& \phi^{\prime \prime}+\frac{N t}{N b} \theta^{\prime \prime}+\frac{3}{4} \operatorname{Lnf} \phi^{\prime}=0
\end{aligned}
$$

subject to the following boundary conditions:

$$
\begin{aligned}
& f(\eta)=0, f^{\prime}(\eta)=0, \theta^{\prime}(\eta)=-N c[1-\theta(\eta)], \phi(\eta)=1, \quad \text { at } \quad \eta=0 \\
& f^{\prime}(\eta)=0, \theta(\eta)=0, \phi(\eta)=0, \quad \text { as } \quad \eta \rightarrow \infty
\end{aligned}
$$

where $N c$ (convective heating parameter) appearing in boundary condition (35) is defined as:

$$
N c=\frac{h_{f} x^{1 / 4}}{k}\left[\frac{v \alpha_{m}}{\left(1-\hat{\phi}_{\infty}\right) g_{e} \beta\left(T_{f}-T_{\infty}\right)}\right]^{1 / 4}
$$

\section{Nusselt and Sherwood number evaluation}

The understanding of heat and mass transfer at the wall plays an important role in estimating the performance of several microfluidic/ nanofluidic/ thermal devices. The related information with the variation in the properties of wall yields information which may lead to a change in the design with an improvement in the performance and efficiency of the devices. Thus the heat and mass transfer rates are the important characteristics that need to be computed [25]. These quantities, local Nusselt number $N u_{x}$ and the local nanofluid Sherwood number $S h_{x, n}$, can be written as:

$$
N u_{x}=\frac{x q_{w}}{k\left(T_{f}-T_{\infty}\right)}, S h_{x, n}=\frac{x q_{n p}}{D_{B}\left(\hat{\phi}_{w}-\hat{\phi}_{\infty}\right)},
$$

where $q_{w}$ and $q_{n p}$ are the wall heat and nano mass fluxes, respectively.

The modified Nusselt number Nur and modified nanoparticle Sherwood number Shrn can be introduced and represented as follows:

$$
N u r=R a_{x}^{1 / 4} N u_{x}=-\theta^{\prime}(0), \operatorname{Shr} n=R a_{x}^{1 / 4} S h_{x, n}=-\phi^{\prime}(0),
$$




\section{Numerical Solution with Finite Element Solution}

In this section, the effects of important parameters on flow analysis and on heat and mass transfer characteristics are discussed in the form of numerical solution of Eqs. (31)-(33). It's very difficult to find the analytical solution of Eqs. (31)-(33). Hence, the conventional finite element method (FEM), which is a numerical approach, is used as it is the most adaptive and popular method for solving differential equations. The basic step of FEM requires the division of the whole domain into smaller, non-overlapping sub-domains in order to solve the flow physics within the domain geometry. This results in the generation of a grid of elements overlaying the whole domain geometry. It is an enormously useful method (in terms of both resolving material nonlinearity and complex geometrical) and has received significant attention in nonlinear problems involving heat transfer [3,10], nanofluid mechanics [11], membrane structural mechanics [12], biological systems [13], electrical systems [19], and many others. The non-linear coupled differential equations (31)-(33) subject to the boundary conditions (34), (35) have been solved using Finite element method (FEM). By assuming

$$
f^{\prime}=h
$$

The system of Eqs. (31)-(33) can be reduced into a pair of lower order equations as follows:

$$
\begin{aligned}
& h^{\prime \prime}+\frac{1}{4 P r}\left(3 f h^{\prime}-2 h^{2}\right)+(\theta-N r \phi) \cos \delta-M h=0 \\
& \theta^{\prime \prime}+\frac{3}{4} f \theta^{\prime}+N b \phi^{\prime} \theta^{\prime}+N t\left(\theta^{\prime}\right)^{2}=0 \\
& \phi^{\prime \prime}+\frac{N t}{N b} \theta^{\prime \prime}+\frac{3}{4} \operatorname{Lnf} \phi^{\prime}=0
\end{aligned}
$$

The corresponding boundary conditions now become;

$$
\begin{aligned}
f(0) & =0, h(0)=0, \phi(0)=1, \theta^{\prime}(0)=-N c[1-\theta(0)] \text { as } \eta=0 \\
h(\infty) & =0, \theta(\infty)=0, \phi(\infty)=0 \quad \text { as } \quad \eta \rightarrow \infty
\end{aligned}
$$

\section{Variational Formulation}

The variational form associated with Eqs. (40)-(42) over a typical linear element $\left(\eta_{e}, \eta_{e+1}\right)$ is given by

$$
\begin{aligned}
& \int_{\eta_{e}}^{\eta_{e+1}} W_{1}\left\{f^{\prime}-h\right\} d \eta=0 \\
& \int_{\eta_{e}}^{\eta_{e+1}} W_{2}\left\{h^{\prime \prime}+\frac{1}{4 P r}\left(3 f h^{\prime}-2 h^{2}\right)+(\theta-N r \phi) \cos \delta-M h\right\} d \eta=0 \\
& \int_{\eta_{e}}^{\eta_{e+1}} W_{3}\left\{\theta^{\prime \prime}+\frac{3}{4} f \theta^{\prime}+N b \phi^{\prime} \theta^{\prime}+N t\left(\theta^{\prime}\right)^{2}\right\} d \eta=0 \\
& \int_{\eta_{e}}^{\eta_{e+1}} W_{4}\left\{\phi^{\prime \prime}+\frac{N t}{N b} \theta^{\prime \prime}+\frac{3}{4} \operatorname{Lnf} \phi^{\prime}\right\} d \eta=0
\end{aligned}
$$

where $W_{1}, W_{2}, W_{3}$ and $W_{4}$ are arbitrary test function and may be viewed as the variation in $f, h, \theta$ and $\phi$, respectively. 


\section{Finite Element Formulation}

Let the domain be divided into linear elements $\left(\Omega_{e}\right)$. The finite element model can be obtained from Eqs. (45-48) by substituting the approximations of the form

$$
\Theta=\sum_{i=1}^{2} \Theta_{j} \psi_{j}
$$

where, $\Theta$ stands for either $f, h, \theta$, or $\phi$. So,

$$
f=\sum_{j=1}^{2} f_{j} \psi_{j}, h=\sum_{j=1}^{2} h_{j} \psi_{j}, \theta=\sum_{j=1}^{2} \theta_{j} \psi_{j}, \phi=\sum_{j=1}^{2} \phi_{j} \psi_{j}
$$

with $W_{1}=W_{2}=W_{3}=W_{4}=\psi_{j},(j=1,2)$ where $\psi_{j}$ are the linear interpolation functions for a linear element $\Omega_{e}$.

The finite element model of the equations thus formed, is given by:

$$
\left[\begin{array}{l}
{\left[K^{11}\right]\left[K^{12}\right]\left[K^{13}\right]\left[K^{14}\right]} \\
{\left[K^{21}\right]\left[K^{22}\right]\left[K^{23}\right]\left[K^{24}\right]} \\
{\left[K^{31}\right]\left[K^{32}\right]\left[K^{33}\right]\left[K^{34}\right]} \\
{\left[K^{41}\right]\left[K^{42}\right]\left[K^{43}\right]\left[K^{44}\right]}
\end{array}\right]\left[\begin{array}{l}
\{f\} \\
\{h\} \\
\{\theta\} \\
\{\phi\}
\end{array}\right]=\left[\begin{array}{l}
\left\{b^{1}\right\} \\
\left\{b^{2}\right\} \\
\left\{b^{3}\right\} \\
\left\{b^{4}\right\}
\end{array}\right]
$$

where $\left[K^{m n}\right]$ and $\left[b^{m n}\right](m, n=1,2,3,4)$ are the matrices of order $2 \times 2$ and $2 \times 1$ respectively and therefore each element matrix is of order $8 \times 8$. These matrices are defined as follows:

$$
\begin{aligned}
K_{i j}^{11} & =\int_{\eta_{e}}^{\eta_{e+1}} \psi_{i} \frac{\partial \psi_{j}}{\partial \eta} d \eta, \quad K_{i j}^{12}=-\int_{\eta_{e}}^{\eta_{e+1}} \psi_{i} \psi_{j} d \eta, \quad K_{i j}^{13}=K_{i j}^{14}=0, \\
K_{i j}^{22} & =-\int_{\eta_{e}}^{\eta_{e+1}} \frac{\partial \psi_{i}}{\partial \eta} \frac{\partial \psi_{j}}{\partial \eta} d \eta-\frac{1}{2 P r} \int_{\eta_{e}}^{\eta_{e+1}} \psi_{i} \bar{h} \psi_{j} d \eta-M \int_{\eta_{e}}^{\eta_{e+1}} \psi_{i} \psi_{j} d \eta, \\
K_{i j}^{21} & =\frac{3}{4 P r} \int_{\eta_{e}}^{\eta_{e+1}} \psi_{i} \bar{h}^{\prime} \psi_{j} d \eta, \quad K_{i j}^{23}=\cos \delta \int_{\eta_{e}}^{\eta_{e+1}} \psi_{i} \psi_{j} d \eta, \\
K_{i j}^{24} & =-N r \cos \delta \int_{\eta_{e}}^{\eta_{e+1}} \psi_{i} \psi_{j} d \eta, K_{i j}^{31}=\frac{3}{4} \int_{\eta_{e}}^{\eta_{e+1}} \psi_{i} \bar{\theta}^{\prime} \psi_{j} d \eta, \quad K_{i j}^{32}=0, \\
K_{i j}^{33} & =-\int_{\eta_{e}}^{\eta_{e+1}} \frac{\partial \psi_{i}}{\partial \eta} \frac{\partial \psi_{j}}{\partial \eta} d \eta+N t \int_{\eta_{e}}^{\eta_{e+1}} \psi_{i} \bar{\theta}^{\prime} \frac{\partial \psi_{i}}{\partial \eta} d \eta, \\
K_{i j}^{34} & =N b \int_{\eta_{e}}^{\eta_{e+1}} \psi_{i} \overline{\theta^{\prime}} \frac{\partial \psi_{i}}{\partial \eta} d \eta, \quad K_{i j}^{42}=0, \quad K_{i j}^{41}=\frac{3}{4} L n \int_{\eta_{e}}^{\eta_{e+1}} \psi_{i} \bar{\phi}^{\prime} \frac{\partial \psi_{i}}{\partial \eta} d \eta, \\
K_{i j}^{43} & =-\frac{N t}{N b} \int_{\eta_{e}}^{\eta_{e+1}} \frac{\partial \psi_{i}}{\partial \eta} \frac{\partial \psi_{j}}{\partial \eta} d \eta, \quad K_{i j}^{44}=-\int_{\eta_{e}}^{\eta_{e+1}} \frac{\partial \psi_{i}}{\partial \eta} \frac{\partial \psi_{j}}{\partial \eta} d \eta,
\end{aligned}
$$

where

$$
\bar{h}=\sum_{i=1}^{2} \overline{h_{i}} \psi_{i}, \overline{h^{\prime}}=\sum_{i=1}^{2} \overline{h_{i}} \frac{\partial \psi_{i}}{\partial \eta}, \overline{\theta^{\prime}}=\sum_{i=1}^{2} \overline{\theta_{i}} \frac{\partial \psi_{i}}{\partial \eta}, \overline{\phi^{\prime}}=\sum_{i=1}^{2} \overline{\phi_{i}} \frac{\partial \psi_{i}}{\partial \eta}
$$

The computational domain is discretized with uniformly distributed 2000 linear elements. The length of the boundary layer region i.e. $\eta_{\infty}$ is chosen as 14 . Results were obtained even for large values of $\eta_{\infty}$, but after $\eta_{\infty}=14$, no appreciable effect on results was observed. Therefore, the boundary layer thickness is chosen as 14 . At every node four functions $f, f^{\prime}, \theta$ 
Table 1 Comparison of $N u r$ of regular fluid for various values of $\operatorname{Pr}$ with $L n=10, N b=N t=N r=10^{-5}$, $M=0$

\begin{tabular}{lllll}
\hline $\operatorname{Pr}$ & Bejan [5] & Kuznetsov and Nield [17] & Narahari et al. [19] & Present results \\
\hline 1 & 0.401 & 0.401 & 0.401 & 0.4014 \\
10 & 0.465 & 0.463 & 0.459 & 0.4654 \\
100 & 0.490 & 0.481 & 0.473 & 0.4904 \\
1000 & 0.499 & 0.484 & 0.474 & 0.4970 \\
\hline
\end{tabular}

and $\phi$ are to be calculated; hence after assembly of the element equations, we obtain a system of 8004 non-linear equations.

Owing to the nonlinearity of the system an iterative scheme has been used to solve it iteratively. The system of equations is linearized by incorporating known functions $\bar{f}, \bar{f}^{\prime}, \bar{\theta}, \bar{\phi}$ which are calculated using the approximate values of variables $f, f^{\prime}, \theta, \phi$ at node $i$ on previous iteration, as given in Eq. (50). The whole system is solved by using a Gaussian elimination method and the whole procedure is executed in MATLAB. This gives a new set of values of unknowns $f, f^{\prime}, \theta, \phi$ and the process continues until the required accuracy of $1 \times 10^{-5}$ is achieved.

\section{Validation of the Numerical Procedure}

For validation purpose, results were compared with previously reported results in the literature. The results for the regular fluid at different values of $\mathrm{Pr}$ were compared with those reported by Bejan [5], Kuznetsov and Nield [17] and Narahari et al. [19], has been captured in Table 1. Closer correlation has been achieved as compared to results computed by $[5,17,19]$. On the other hand, Table 2 shows the excellent correlation between the current FEM computation and the earlier results of Aziz and Khan [4] on the modified Nusselt and nanoparticle Sherwood number under the influence of the various parameters such as $\mathrm{Nb}, \mathrm{Nr}, \mathrm{Pr}$.

\section{Double Mesh Principle}

To estimate the error and compute the rate of convergence in the computed numerical solution, the double mesh principle was used $[18,21,24]$. As the exact solution of the problem was unknown, and therefore to approximate the pointwise errors $\left|(\tilde{\Theta}-\Theta)\left(\eta_{i}\right)\right|$; $i=0,1,2, \ldots N$, we have used variant of double mesh principle, where $\Theta(\eta)$ and $\tilde{\Theta}(\eta)$ denote the numerical solutions of the system of ODE's (39)-(42) at the two consecutive different mesh. A numerical solution $\Theta(\eta)$ to $\tilde{\Theta}(\eta)$ is calculated which was given by FEM on the mesh $\left\{\hat{\eta}_{i}\right\}$ that contained the mesh points $\eta_{i}$ of the original mesh and their midpoints (i.e. $\hat{\eta}_{2 i}=\eta_{i}, i=0,1, \ldots, N, \hat{\eta}_{2 i+1}=\frac{\eta_{i}+\eta_{i+1}}{2}, i=0,1, \ldots, N-1$ ). Then at the mesh points, $\eta_{i}, i=0,1, \ldots, N$, the maximum error is computed as:

$$
E_{N}=\max _{0 \leq i \leq N}\left|\Theta\left(\eta_{i}\right)-\tilde{\Theta}\left(\eta_{i}\right)\right|
$$




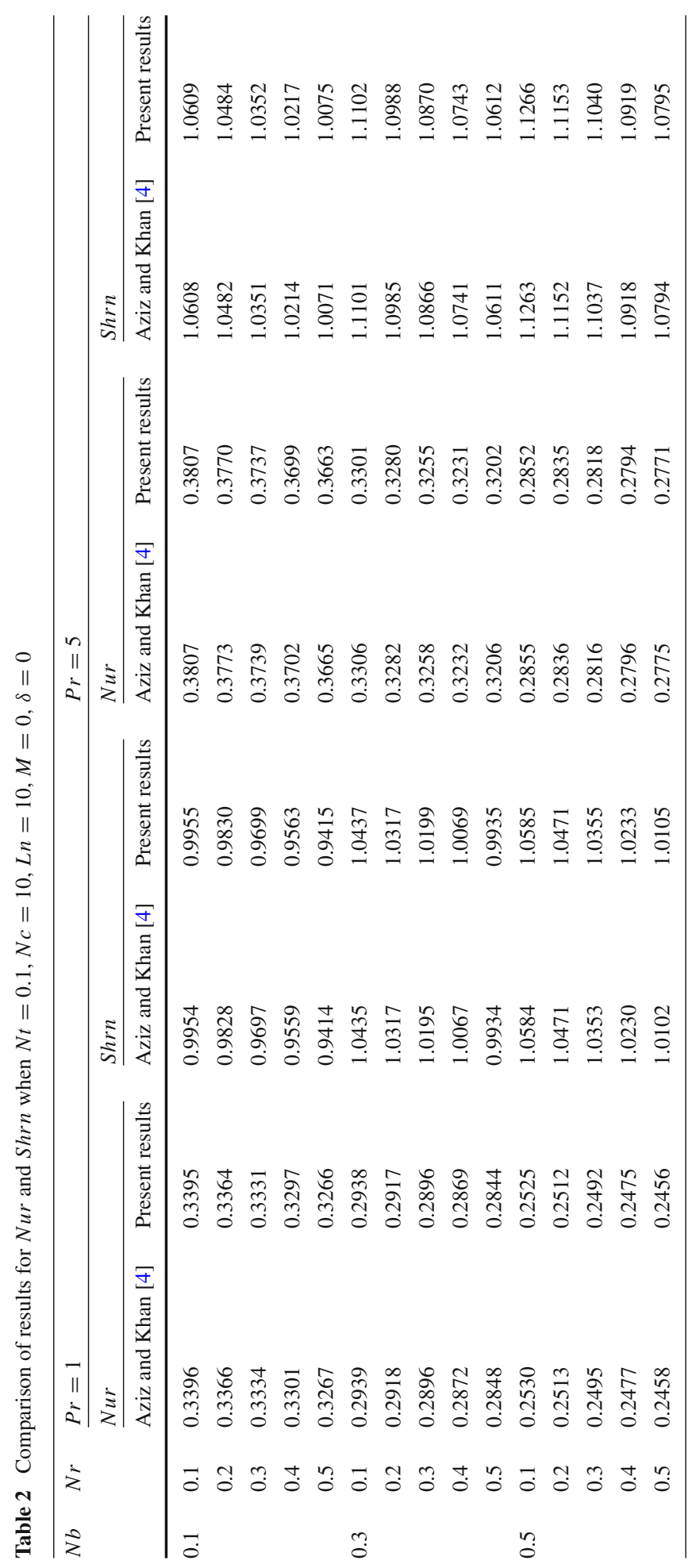


Table 3 Errors and rate of convergence for the finite element method (FEM)

\begin{tabular}{|c|c|c|c|c|c|c|}
\hline \multirow[t]{2}{*}{$h_{e}$} & \multicolumn{2}{|l|}{$f^{\prime}$} & \multicolumn{2}{|l|}{$\underline{\theta}$} & \multicolumn{2}{|l|}{$\underline{\phi}$} \\
\hline & Error & Rate & Error & Rate & Error & Rate \\
\hline 0.128 & $2.0186 \mathrm{e}-2$ & 1.1664 & $7.0795 \mathrm{e}-2$ & 1.5609 & $4.0550 \mathrm{e}-2$ & 1.4903 \\
\hline 0.064 & $8.9935 \mathrm{e}-3$ & 1.2898 & $2.3994 \mathrm{e}-2$ & 1.6565 & $1.4433 \mathrm{e}-2$ & 1.5759 \\
\hline 0.032 & $3.6779 \mathrm{e}-3$ & 1.4059 & $7.6112 \mathrm{e}-3$ & 1.7482 & $4.8414 \mathrm{e}-3$ & 1.6549 \\
\hline 0.016 & $1.3880 \mathrm{e}-3$ & 1.5135 & $2.2656 \mathrm{e}-3$ & 1.8108 & $1.5374 \mathrm{e}-3$ & 1.7238 \\
\hline 0.008 & $4.8616 \mathrm{e}-4$ & 1.5849 & $6.4578 \mathrm{e}-4$ & 1.9468 & $4.6547 \mathrm{e}-4$ & 1.8136 \\
\hline 0.004 & $1.6206 \mathrm{e}-4$ & 1.6328 & $1.6751 \mathrm{e}-4$ & 2.0004 & $1.3242 \mathrm{e}-4$ & 1.9091 \\
\hline 0.002 & $5.2259 \mathrm{e}-5$ & - & $4.1868 \mathrm{e}-5$ & - & $3.5258 \mathrm{e}-5$ & - \\
\hline
\end{tabular}

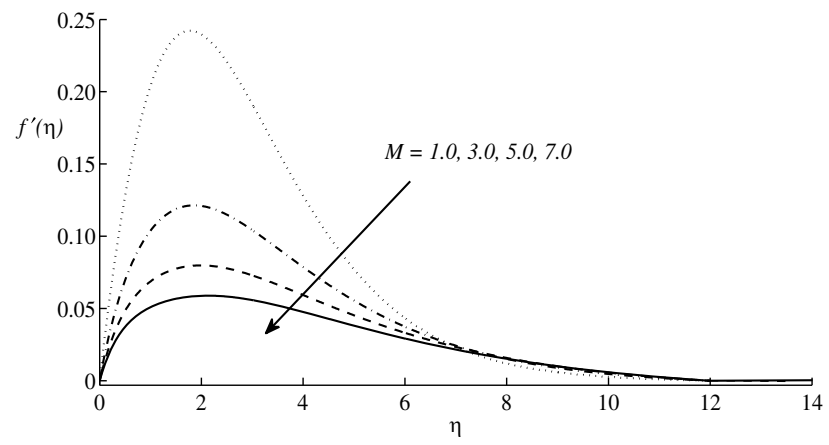

Fig. 2 Effect of magnetic field parameter on velocity profile $f^{\prime}(\eta)$

From these estimates of the errors, the corresponding order of convergence has been obtained, which is defined as:

$$
r^{N}=\log _{2} \frac{E_{N}}{E_{2 N}}
$$

From Table 3, it is concluded that at each step, i.e after each refinement, approximate error corresponding to each function is reducing. Hence, the approximated solution of the current problem is approaching the exact solution. Also, an increment in the convergence rate is observed at each step, which shows that the computed numerical solution is rapidly converging on the exact solution.

\section{Computations and Discussion}

Numerical computations have been carried out for different values of the parameters involved, namely, $\mathrm{M}, \mathrm{Nr}, \mathrm{Nc}, \mathrm{Pr}, \mathrm{Ln}, \mathrm{Nb}, \mathrm{Nt}$ that describe the flow characteristics, heat and mass transfer and the results are reported in terms of graphs and tables. In Figs. 2, 3, 4, 5, 6, 7, 8, 9, 10, 11, 12, 13, 14, 15, 16, 17, 18 and 19, we generally utilize the following data (unless otherwise stated): $P r=5, N b=N r=N c=0.5, N t=0.3$, $L n=10, M=1, \delta=\pi / 4$.

Table 4 indicates dependency of the modified Nusselt number $N u r$ and modified nanoparticle Sherwood number Shrn over changes in the Prandtl number Pr, the Brownian motion 


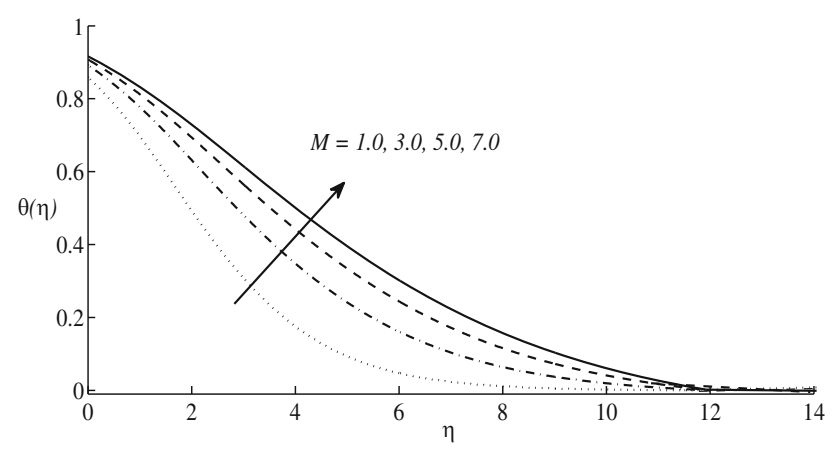

Fig. 3 Effect of magnetic field parameter on temperature profile $\theta(\eta)$

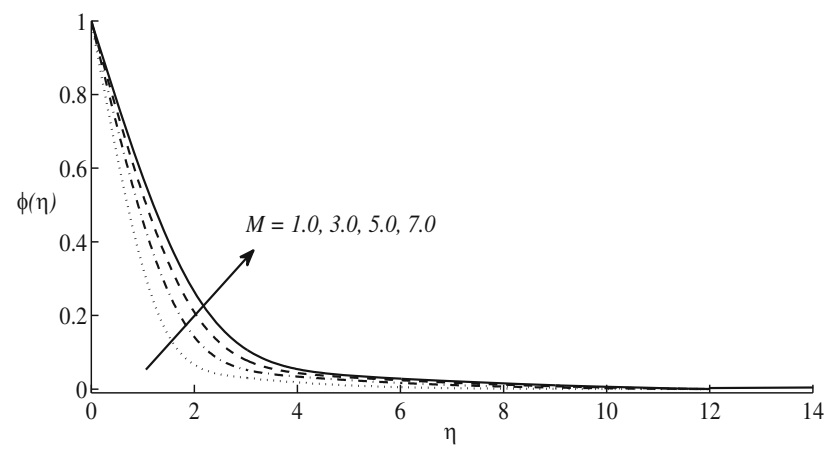

Fig. 4 Effect of magnetic field parameter on nanoparticle volume fraction profile $\phi(\eta)$

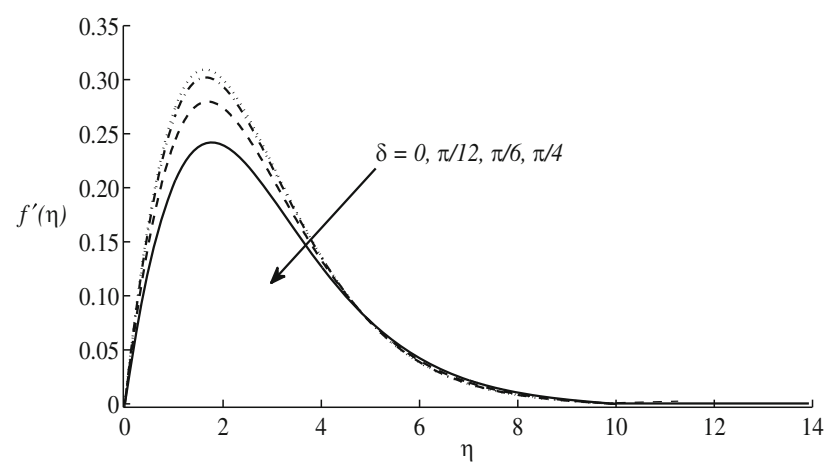

Fig. 5 Effect of angle of inclination on velocity profile $f^{\prime}(\eta)$

parameter $\mathrm{Nb}$ and the buoyancy parameter $\mathrm{Nr}$ when the rest of the parameters are fixed. The modified Nusselt and nanoparticle Sherwood numbers increase with an increase in the Prandtl number $\mathrm{Pr}$. For a fixed $\mathrm{Pr}$, both $\mathrm{Nur}$ and $\mathrm{Shrn}$ decrease as $\mathrm{Nb}$ and $\mathrm{Nr}$ increase. Table 5 shows the changes in the magnetic field parameter $M$, the thermophoretic parameter $N t$, and the angle of inclination $\delta$ affect the modified Nusselt number and the modified nanofluid Sherwood number. It is noticed that the performance of heat and nanoparticle mass transfer of the plate decrease as the magnetic field strength , $M$, and angle of inclination, $\delta$, are 


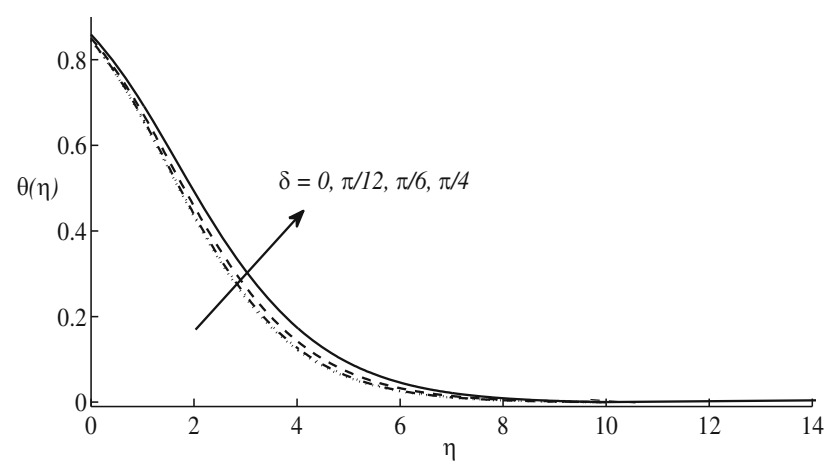

Fig. 6 Effect of angle of inclination on temperature profile $\theta(\eta)$

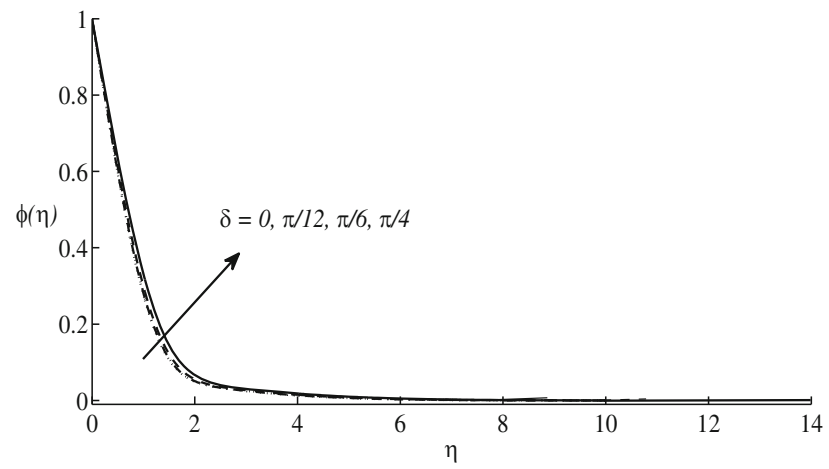

Fig. 7 Effect of angle of inclination on nanoparticle volume fraction profile $\phi(\eta)$

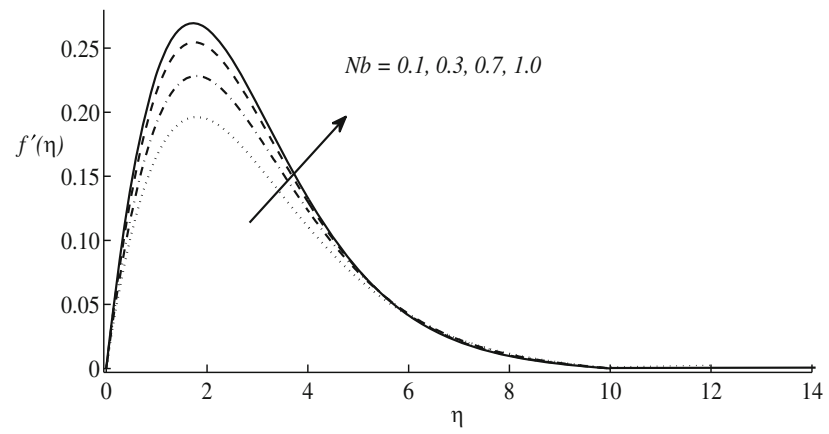

Fig. 8 Effect of Brownian motion parameter on velocity profile $f^{\prime}(\eta)$

gradually enlarged. In the same table, the corresponding heat and nanoparticle mass transfer are also represented for different values of $N t$. The effect of the nanofluid Lewis number on the the modified Nusselt number and the modified nanoparticle Sherwood number is shown in Table 6. As the nanofluid Lewis number increases, the modified Nusselt number decreases slightly but there is a substantial increase in the modified nanoparticle Sherwood number. Tables 4, 5 and 6 provide information about the heat and mass transfer characteristics of the flow in a form convenient for research and engineering calculations. 


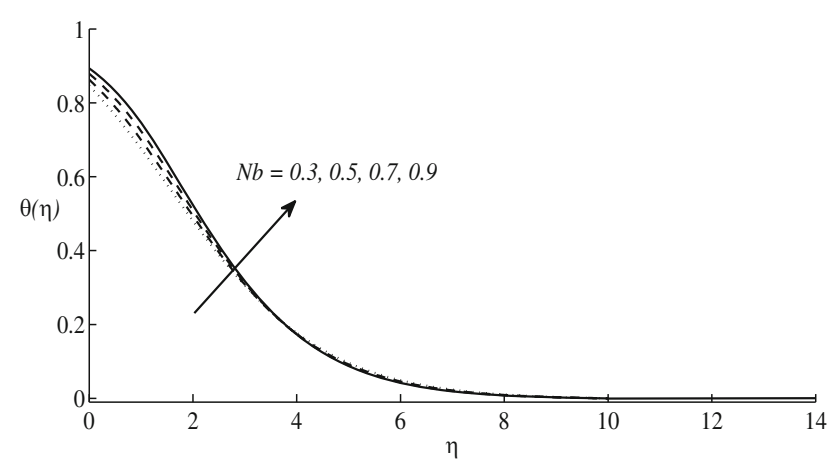

Fig. 9 Effect of Brownian motion parameter on temperature profile $\theta(\eta)$

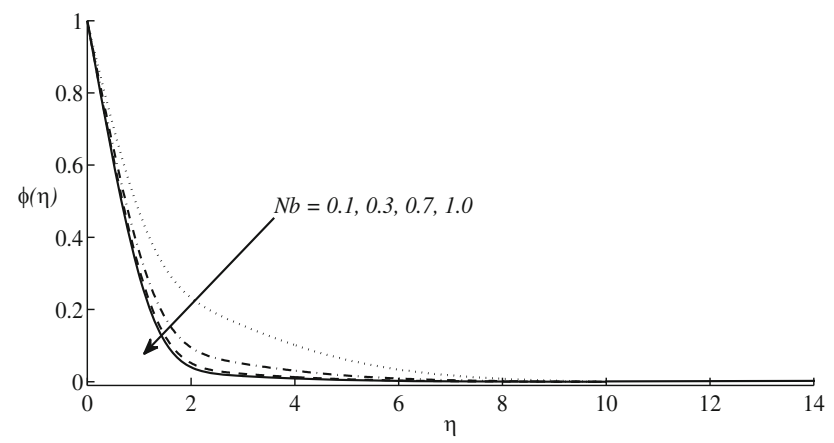

Fig. 10 Effect of Brownian motion parameter on nanoparticle volume fraction profile $\phi(\eta)$

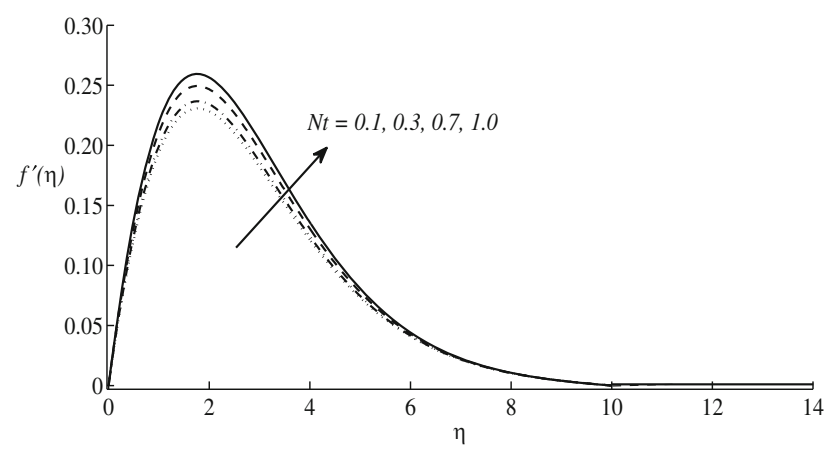

Fig. 11 Effect of thermophoresis parameter on velocity profile $f^{\prime}(\eta)$

Figures 2, 3, and 4 elucidate the variations of the functions $f^{\prime}(\eta), \theta(\eta)$ and $\phi(\eta)$ under the influence of the magnetic field parameter $M$. It is clearly observed that the velocity of the fluid decreases, whereas the temperature increases, with increasing strength of magnetic field. As the application of a transverse magnetic field will result a resistive/drag force, known as Lorentz force, which tends to resist fluid flow and as a result this force prevents the development of momentum and decelerates the flow. Against the action of magnetic field, the additional work done in dragging the nanofluid is expressed as thermal energy. This heats the nanofluid and increases temperature. Consequently, the presence of a magnetic field 


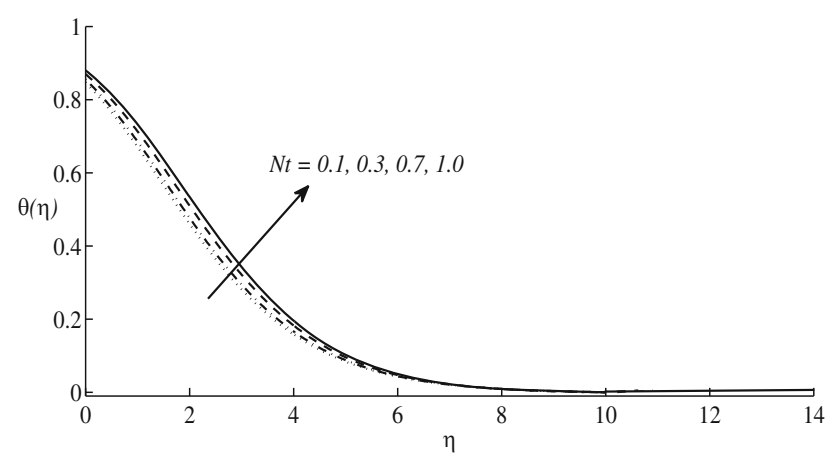

Fig. 12 Effect of thermophoresis parameter on temperature profile $\theta(\eta)$

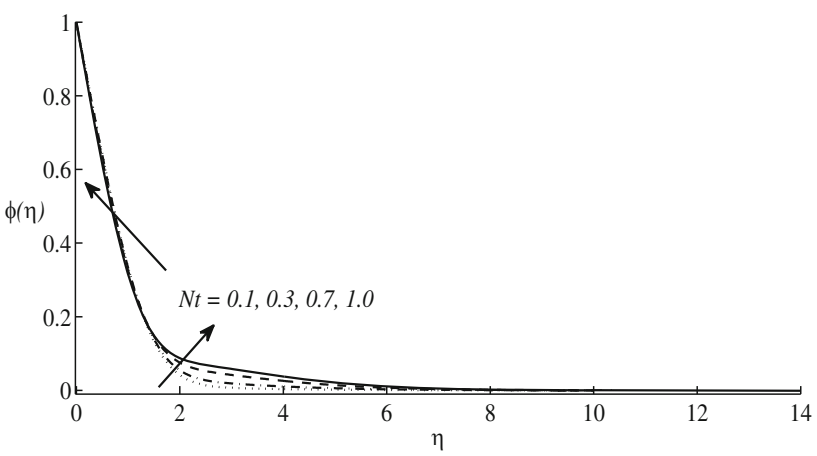

Fig. 13 Effect of thermophoresis parameter on nanoparticle volume fraction profile $\phi(\eta)$

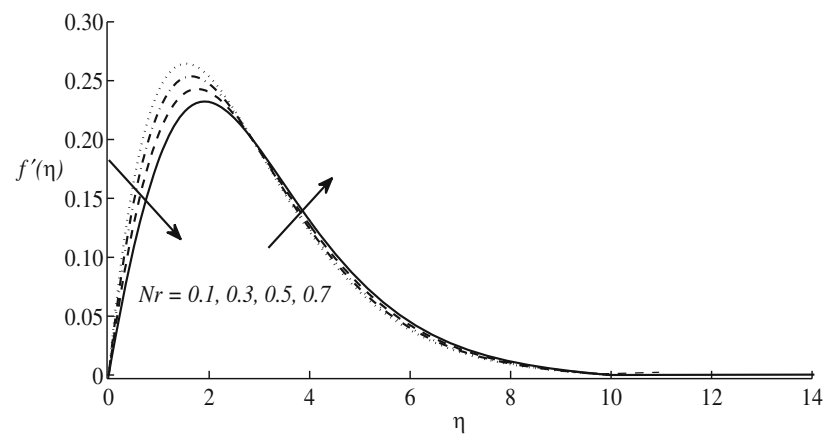

Fig. 14 Effect of buoyancy ratio parameter on velocity profile $f^{\prime}(\eta)$

attenuates the thickness of momentum boundary layer and augments the thickness of thermal boundary layer. Moreover, the warming of the boundary layer also helps in nanoparticle diffusion due to which a rise in nanoparticle volume fraction $\phi(\eta)$ can be observed, as shown in Fig. 4.

In Figs. 5, 6 and 7, the influence of the plate inclination from the vertical, $\delta$, ranging from 0 to $\pi / 4$, on the velocity $f^{\prime}(\eta)$, temperature $\theta(\eta)$ and nanoparticle volume fraction $\phi(\eta)$ profiles are depicted, respectively. It is observed from Fig. 5 that within the hydrodynamic boundary layer, the velocity of the fluid is diminished with an augmenta- 


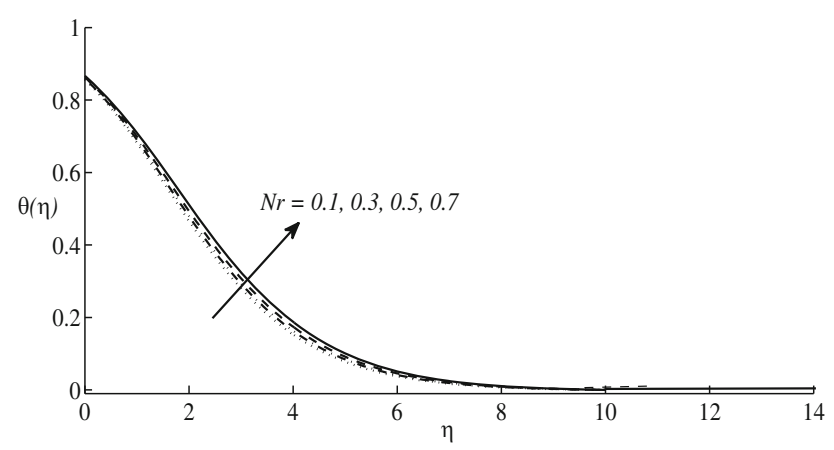

Fig. 15 Effect of buoyancy ratio parameter on temperature profile $\theta(\eta)$

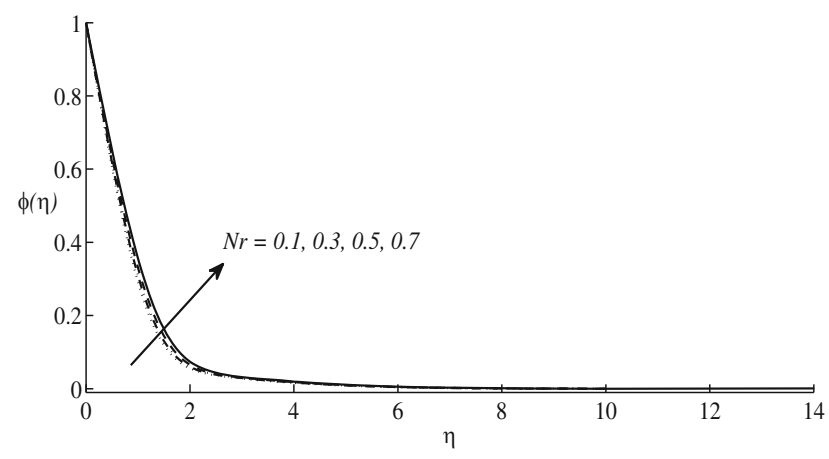

Fig. 16 Effect of buoyancy ratio parameter on nanoparticle volume fraction profile $\phi(\eta)$

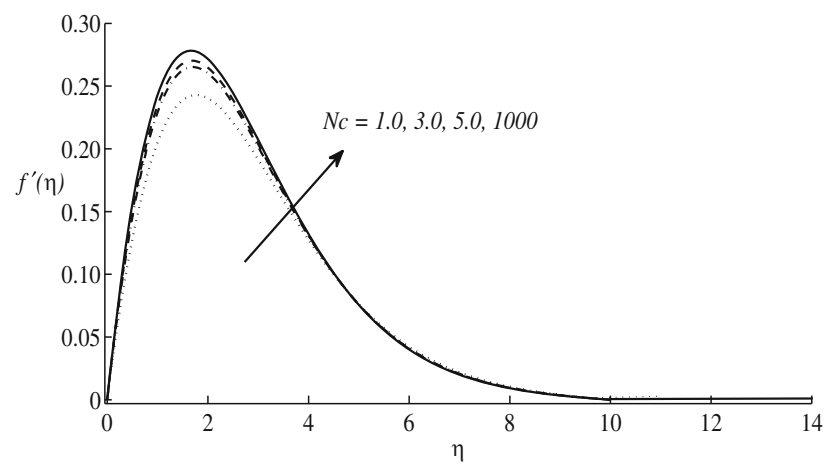

Fig. 17 Effect of convective heating parameter on velocity profile $f^{\prime}(\eta)$

tion of inclination angle. This is because to the plate's alignment via the thermal buoyancy term, $g\left[-\left(\rho_{p}-\rho_{f_{\infty}}\right)\left(\hat{\phi}-\hat{\phi}_{\infty}\right)+\left(1-\hat{\phi}_{\infty}\right) \rho_{f_{\infty}} \beta\left(T-T_{\infty}\right)\right] \cos \delta$, which is arising in the momentum equation (2). As the value of $\delta$ increases, the corresponding value of $\cos \delta$ decreases. This causes the buoyancy effect to be vanished with increasing the plate inclination. Consequently, the driving force to the fluid attenuates, resulting in decrease of velocity of the fluid. A similar type of trend has been found by Alam et al. [1] in case of velocity 


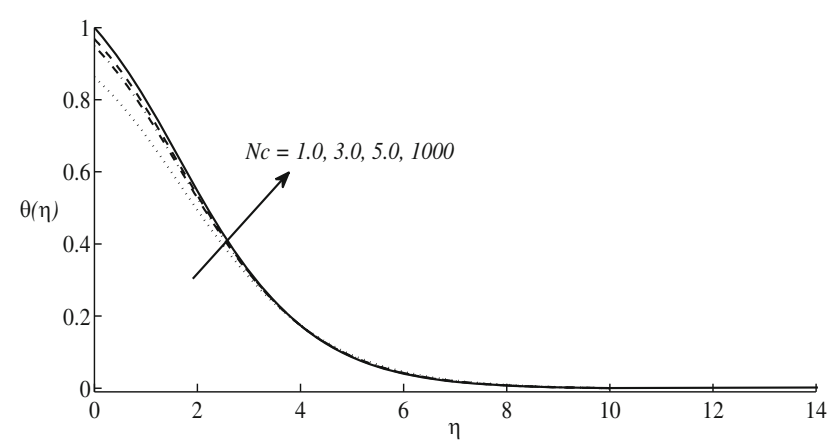

Fig. 18 Effect of convective heating parameter on temperature profile $\theta(\eta)$

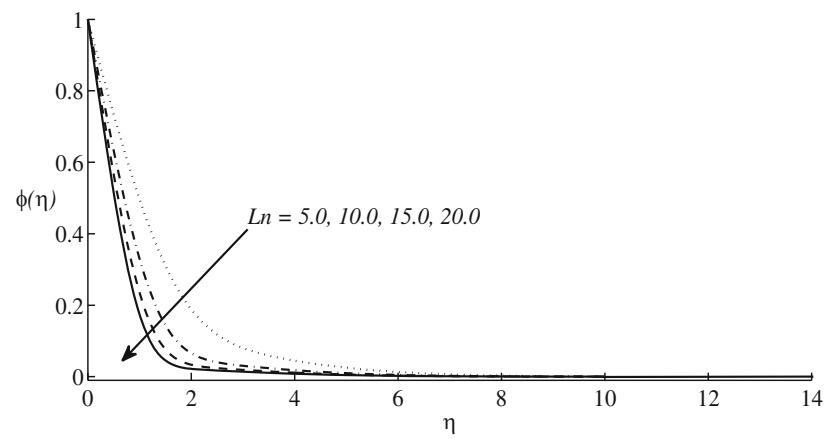

Fig. 19 Effect of nanofluid Lewis parameter on nanoparticle volume fraction profile $\phi(\eta)$

profile. On the other hand, a depletion in buoyancy effect will enhance thermal and species (nanoparticle) diffusion, which is shown in Figs. 6 and 7.

Brownian motion is the haphazard motion of nanoparticles inside the base fluid due to of the continuous collision of nanoparticle with the molecules of base fluid. This motion of the particles is described by parameter $N b$, known as Brownian motion coefficient. Figures 8, 9 and 10 illustrate the effect of $N b$ on velocity $f^{\prime}(\eta)$, temperature $\theta(\eta)$ and concentration $\phi(\eta)$ profiles. With an increase in $\mathrm{Nb}$, the randomness of the nanoparticles increased and as a result, nanoparticles move more chaotically, causing more collisions in the system and vice versa. This increase in number of collisions and velocity result an increase in heat transfer properties, and thus, the value of temperature increases. Simultaneously, the increase in $\mathrm{Nb}$ has an adverse effect on the concentration of nanoparticles along the wall. The nanoparticles start moving away from the boundary into the fluid by increasing the random motion of nanoparticles which causing a decrease in the value of concentration of nanoparticles along the wall.

The phenomenon of diffusion of particles, in the presence of temperature gradient, is known as thermophoresis. The variation of velocity $f^{\prime}(\eta)$, temperature $\theta(\eta)$ and nanoparticle concentration $\phi(\eta)$ for various values of $N t$ is depicted in Figs. 11, 12 and 13. Augmentation in the value of $N t$ causes temperature gradient which results in escalating the force (thermophoretic) between nanoparticles. This force is responsible for more fluid being heated and elevates the temperature. The same effect is observed in the case of nanoparticle concentration by strengthening the effect of thermophoresis $N t$, as shown in Fig. 13. 
Table 4 Variation of Nur and $\mathrm{Shrn}$ for $\mathrm{Pr}, \mathrm{Nb}$ and $\mathrm{Nr}$ for $\delta=\pi / 4, N c=10, N t=0.5$, $M=0.1, L n=10$

\begin{tabular}{|c|c|c|c|c|c|c|c|}
\hline \multirow[t]{2}{*}{$\mathrm{Nb}$} & \multirow[t]{2}{*}{$N r$} & \multicolumn{2}{|l|}{$\underline{P r}=1$} & \multicolumn{2}{|l|}{$P r=5$} & \multicolumn{2}{|c|}{$\operatorname{Pr}=10$} \\
\hline & & Nur & Shrn & Nur & Shrn & Nur & Shrn \\
\hline \multirow[t]{6}{*}{0.1} & 0.1 & 0.2120 & 0.7253 & 0.2183 & 0.7418 & 0.2191 & 0.7441 \\
\hline & 0.2 & 0.2076 & 0.7094 & 0.2136 & 0.7252 & 0.2144 & 0.7273 \\
\hline & 0.3 & 0.2030 & 0.6926 & 0.2086 & 0.7076 & 0.2094 & 0.7097 \\
\hline & 0.4 & 0.1981 & 0.6748 & 0.2034 & 0.6890 & 0.2042 & 0.6909 \\
\hline & 0.5 & 0.1929 & 0.6559 & 0.1979 & 0.6692 & 0.1986 & 0.6710 \\
\hline & 0.6 & 0 . & 0 & 0 & 0 . & 7 & 196 \\
\hline \multirow[t]{6}{*}{0.3} & 0.1 & 0.1840 & 0.8280 & 0.1897 & 0.8420 & 0.1905 & 0.8439 \\
\hline & 0.2 & 0.18 & 0.8 & 0 & 0 . & 19 & 0.8299 \\
\hline & 0.3 & 0.1791 & 0.8000 & 0.1845 & 0.8 & 52 & 0.8151 \\
\hline & 0.4 & 0.1766 & 0.7849 & 0 . & 0.7 & 325 & 0.7995 \\
\hline & 0.5 & 0.17 & 0.76 & 0.17 & 0 & 6 & 0.7 \\
\hline & 0.6 & 0.1710 & 0.7519 & 0.1758 & 0.7640 & 0.1765 & 0.7656 \\
\hline \multirow[t]{6}{*}{0.5} & 0.1 & 0.1581 & 0.8502 & 0.1632 & 0.8639 & 0.1639 & 0.8658 \\
\hline & 0.2 & 0.1564 & 0.8371 & 0.1614 & 0.8506 & 0.1620 & 0.8524 \\
\hline & 0.3 & 0.1546 & 0.8235 & 0.1594 & 0.8366 & 0.1601 & 0.8384 \\
\hline & 0.4 & 0.1527 & 0.8091 & 0.1574 & 0.8219 & 580 & 0.8236 \\
\hline & 0.5 & 0 . & 0.7939 & 0.1552 & 0.8064 & 0.1559 & 0.8081 \\
\hline & 0.6 & 0.1486 & 0.7778 & 0.1530 & 0.7900 & 0.1536 & 0.7916 \\
\hline
\end{tabular}

Table 5 Variation of $N u r$ and Shrn for $M, N t$ and $\delta$ for $N c=10, P r=5.0, N b=N r=0.5, L n=10$

\begin{tabular}{|c|c|c|c|c|c|c|c|}
\hline \multirow[t]{2}{*}{$N t$} & \multirow[t]{2}{*}{$\delta$} & \multicolumn{2}{|c|}{$M=1.0$} & \multicolumn{2}{|c|}{$M=3.0$} & \multicolumn{2}{|c|}{$M=5.0$} \\
\hline & & Nur & Shrn & Nur & Shrn & Nur & Shrn \\
\hline \multirow[t]{4}{*}{0.1} & 0 & 0.1979 & 0.8541 & 0.1500 & 0.6858 & 0.1266 & 0.5903 \\
\hline & $\pi / 12$ & 0.1956 & 0.8446 & 0.1480 & 0.6768 & 0.1249 & 0.5819 \\
\hline & $\pi / 6$ & 0.1885 & 0.8156 & 0.1418 & 0.6490 & 0.1198 & 0.5561 \\
\hline & $\pi / 4$ & 0.1759 & 0.7636 & 0.1312 & 0.5997 & 0.1111 & 0.5105 \\
\hline \multirow[t]{4}{*}{0.3} & 0 & 0.1829 & 0.8692 & 0.1387 & 0.6958 & 0.1169 & 0.5986 \\
\hline & $\pi / 12$ & 0.1808 & 0.8596 & 0.1368 & 0.6867 & 0.1153 & 0.5901 \\
\hline & $\pi / 6$ & 0.1743 & 0.8300 & 0.1310 & 0.6585 & 0.1105 & 0.5642 \\
\hline & $\pi / 4$ & 0.1626 & 0.7769 & 0.1211 & 0.6086 & 0.1024 & 0.5186 \\
\hline \multirow[t]{4}{*}{0.5} & 0 & 0.1696 & 0.8886 & 0.1284 & 0.7089 & 0.1081 & 0.6097 \\
\hline & $\pi / 12$ & 0.1676 & 0.8787 & 0.1267 & 0.6996 & 0.1067 & 0.6012 \\
\hline & $\pi / 6$ & 0.1615 & 0.8483 & 0.1213 & 0.6709 & 0.1022 & 0.5751 \\
\hline & $\pi / 4$ & 0.1507 & 0.7939 & 0.1121 & 0.6204 & 0.0946 & 0.5294 \\
\hline
\end{tabular}

The buoyancy-ratio parameter $\mathrm{Nr}$ is defined as the ratio of the variation of the fluid density (due to the variation of the concentration) to the variation of the density of the nanofluid (due to the variation of temperature). Figures 14,15 and 16 present the behavior of buoyancy ratio parameter $N r$ on the velocity $f^{\prime}(\eta)$, temperature $\theta(\eta)$ and nanoparticle volume fraction $\phi(\eta)$ profiles. It is observed from these figures that an increase in the Buoyancy-ratio parameter 
Table 6 Variation of Nur and Shrn for $\mathrm{Ln}, \mathrm{Nt}$ and $\mathrm{Nr}$ for $\delta=\pi / 4, N c=10, N b=0.5$, $M=0.1, \operatorname{Pr}=5.0$

\begin{tabular}{|c|c|c|c|c|c|c|c|}
\hline \multirow[t]{2}{*}{$N t$} & \multirow[t]{2}{*}{$\mathrm{Nr}$} & \multicolumn{2}{|l|}{$L n=1$} & \multicolumn{2}{|l|}{$L n=5$} & \multicolumn{2}{|c|}{$\underline{L n}=10$} \\
\hline & & Nur & Shrn & Nur & Shrn & Nur & Shrn \\
\hline \multirow[t]{6}{*}{0.1} & 0.1 & 0.2105 & 0.2710 & 0.1897 & 0.6148 & 0.1839 & 0.8201 \\
\hline & 0.2 & 0.2025 & 0.2607 & 0.1868 & 0.6025 & 0.1821 & 0.8071 \\
\hline & 0.3 & 0.1938 & 0.2495 & 0.1837 & 0.5895 & 0.1801 & 0.7934 \\
\hline & 0.4 & 0.1842 & 0.2374 & 0.1805 & 0.5757 & 0.1781 & 0.7789 \\
\hline & 0.5 & 0.1736 & 0.2241 & 0.1770 & 0.5608 & 0.1759 & 0.7636 \\
\hline & 0.6 & 0.1617 & 0.2095 & 0.1733 & 0.5447 & 0.1736 & 0.7472 \\
\hline \multirow[t]{6}{*}{0.3} & 0.1 & 0.1986 & 0.2437 & 0.1763 & 0.6191 & 0.1704 & 0.8333 \\
\hline & 0.2 & 0.1902 & 0.2336 & 0.1734 & 0.6067 & 0.1686 & 0.8203 \\
\hline & 0.3 & 0.1810 & 0.2228 & 0.1704 & 0.5937 & 0.1667 & 0.8066 \\
\hline & 0.4 & 0.1709 & 0.2116 & 0.1672 & 0.5798 & 0.1647 & 0.7922 \\
\hline & 0.5 & 0.1598 & 0.1999 & 0.1638 & 0.5649 & 0.1626 & 0.7769 \\
\hline & 0.6 & 0.1477 & 0.1879 & 0.1601 & 0.5488 & 0.1604 & 0.7607 \\
\hline \multirow[t]{6}{*}{0.5} & 0.1 & 0.1875 & 0.2235 & 0.1641 & 0.6283 & 0.1581 & 0.8502 \\
\hline & 0.2 & 0.1788 & 0.2133 & 0.1613 & 0.6159 & 0.1564 & 0.8371 \\
\hline & 0.3 & 0.1693 & 0.2031 & 0.1583 & 0.6027 & 0.1546 & 0.8235 \\
\hline & 0.4 & 0.1589 & 0.1930 & 0.1552 & 0.5888 & 0.1527 & 0.8091 \\
\hline & 0.5 & 0.1477 & 0.1836 & 0.1519 & 0.5738 & 0.1507 & 0.7939 \\
\hline & 0.6 & 0.1358 & 0.1754 & 0.1484 & 0.5577 & 0.1486 & 0.7778 \\
\hline
\end{tabular}

increases the magnitude of the dimensionless temperature and nanoparticle concentration while decreases the magnitude of the dimensionless velocity of the nanofluid.

Figures 17 and 18 display the effect of the convective heating parameter $N c$ on velocity $f^{\prime}(\eta)$ and temperature $\theta(\eta)$ profiles. It is noted that velocity and the temperature of the fluid increase with an increase in $N c$. Figure 19 depicts that the nanofluid Lewis number significantly affects the concentration of nanoparticles $\phi(\eta)$. For a base fluid of certain thermal diffusivity $\alpha_{m}$, a higher Lewis implies a lower Brownian diffusion coefficient $D_{B}$ (as $L n=$ $\alpha_{m} / D_{B}$ ) which must result in a shorter penetration depth for the concentration boundary layer. This is exactly what Fig. 19 represents.

\section{Conclusion}

A combined similarity-numerical approach is used to study the natural convective boundary layer flow of a nanofluid past a convectively heated inclined plate in the presence of magnetic field, using a model in which Brownian motion and thermophoresis are accounted for. By use of appropriate similarity transformation, the essential partial differential equations with the corresponding boundary conditions are numerically tackled using Galerkin-finite element method (FEM). The impact of the pertinent parameters upon the flow, temperature, nanoparticle-concentration, modified Nusselt and Sherwood numbers are represented in tabular as well as in graphical form. The use of a convective boundary condition instead of a constant temperature or a constant heat flux makes this approach novel. The computational analysis leads to the following conclusions: 
1. Amplifying the strength of magnetic field $M$ attenuates the thickness of the momentum boundary layer and expands the thermal and nano-volume fraction boundary layer. The application of an external magnetic field produces Lorentz drag force which retards the fluid motion. By customizing the external magnetic field, the transfer of heat can be controlled. In the field of 'smart' cooling devices, widespread growth is based on this idea.

2. With an augmentation in the magnetic parameter $M$, the magnitude of heat and nano-mass transfer rates decrease as a consequence of intensified Lorentz drag force.

3. Strengthening the thermophoresis $N t$ and Brownian motion $N b$ parameters, the rate of heat and nano-mass transfer decrease for an increase in the value of magnetic field parameter $M$. The heat and mass transfer rates can be altered by taking different combinations of base fluid and nanoparticles. This idea can be implemented for numerous industrial applications involving inclined/vertical plates (production of glass fibres, plastic products, tetrapacks etc.) in adjusting the heat transfer rates.

4. With the mounting values of an angle of inclination $\delta$, the width of the momentum boundary layer decays whereas the reverse effect occurs for temperature and concentration boundary layers.

5. The use of a convective boundary condition instead of a constant temperature or a constant heat flux makes this approach novel. As, the convective heating parameter $\mathrm{Nc}$ enhances the rate of heat transfer at the surface of the plate. This effect finds application in case of heat exchangers where the convection in the fluid past the solid surface influenced the conduction in the solid surface.

6. The excellent accuracy of the computed FEM results was shown with the help of double mesh principle.

However, the present study has been focused on the steady-state situation, time-dependent flow of nanofluid over the plate will be addressed in the future investigations. Also the present two-phase model might be extended for turbulent nanofluid flow problem with the inclusion of other slip mechanism viz. diffusiophoresis, inertia and drag force.

Open Access This article is distributed under the terms of the Creative Commons Attribution 4.0 International License (http://creativecommons.org/licenses/by/4.0/), which permits unrestricted use, distribution, and reproduction in any medium, provided you give appropriate credit to the original author(s) and the source, provide a link to the Creative Commons license, and indicate if changes were made.

\section{References}

1. Alam, M., Rahman, M., Sattar, M.: On the effectiveness of viscous dissipation and joule heating on steady magnetohydrodynamic heat and mass transfer flow over an inclined radiate isothermal permeable surface in the presence of thermophoresis. Commun. Nonlinear Sci. Numer. Simul. 14, 2132-2143 (2009)

2. Ali, F., Khan, I., Samiulhaq, S.S.: Conjugate effects of heat and mass transfer on MHD free convection flow over an inclined plate embedded in a porous medium. Abbott D, ed. PLoS ONE 8(6), e65,223 (2013). https://doi.org/10.1371/journal.pone.0065,223

3. Anghel, M., Hossain, M.A., Zeb, S., Pop, I.: Combined heat and mass transfer by free convection past an inclined flat plate. Int. J. Appl. Mech. Eng. 2, 473-497 (2001)

4. Aziz, A., Khan, W.: Natural convective boundary layer flow of a nanofluid past a convectively heated vertical plate. Int. J. Therm. Sci. 52, 83-90 (2012)

5. Bejan, A.: Convection Heat Transfer. Wiley, New York (1984)

6. Buongiorno, J.: Convective transport in nanofluids. J. Heat Transf. 128, 240-250 (2006)

7. Chen, C.H.: Heat and mass transfer in MHD flow by natural convection from a permeable, inclined surface with variable wall temperature and concentration. Acta Mech. 172, 219-235 (2004) 
8. Choi, S.U.S.: Enhancing thermal conductivity of fluids with nanoparticles in developments and applications of non-Newtonian flows. In: Siginer, D.A., Wang, H.P. (eds.) ASME FED 231/MD, vol. 66, pp. 99-105 (1995)

9. Goyal, M., Bhargava, R.: Numerical solution of MHD viscoelastic nanofluid flow over a stretching sheet with partial slip and heat source/sink. ISRN Nanotechnol. 2013, 1-11 (2013)

10. Goyal, M., Bhargava, R.: Boundary layer flow and heat transfer of viscoelastic nanofluids past a stretching sheet with partial slip conditions. Appl. Nanosci. 4, 761-767 (2014a)

11. Goyal, M., Bhargava, R.: Numerical study of thermodiffusion effects on boundary layer flow of nanofluids over a power law stretching sheet. Microfluid. Nanofluidics 17, 591-604 (2014b)

12. Goyal, M., Bhargava, R.: Thermodiffusion effects on boundary layer flow of viscoelastic nanofluids over a stretching sheet with viscous dissipation and non-uniform heat source using hp-finite element method. Proc. IMechE Part N J. Nanoeng. Nanosyst. 230, 124-140 (2014c)

13. Hamad, M.A.A., Pop, I., Ismail, A.I.M.: Magnetic field effects on free convection flow of a nanofluid past a vertical semi-infinite flat plate. Nonlinear Anal. Real World Appl. 12, 1338-1346 (2011)

14. Hatami, M., Jing, D., Song, D., Sheikholeslami, M., Ganji, D.: Heat transfer and flow analysis of nanofluid flow between parallel plates in presence of variable magnetic field using HPM. J. Magn. Magn. Mater. 396, 275-282 (2015)

15. Huminic, G., Huminic, A.: Application of nanofluids in heat exchangers: a review. Renew. Sust. Energy Rev. 16(8), 5625-5638 (2012). https://doi.org/10.1016/j.rser.2012.05.023

16. Khair, K.R., Bejan, A.: Mass transfer to natural convection boundary layer flow driven by heat transfer. Int. J. Heat Mass Transf. 30, 369-376 (1985)

17. Kuznetsov, A., Nield, D.: Natural convective boundary-layer flow of a nanofluid past a vertical plate. Int. J. Therm. Sci. 49, 243-247 (2010)

18. Margenov, S., Vulkov, L., Vulkov, L.G., Wasniewski, J.: Numerical Analysis and Its Applications: 4th International Conference, NAA 2008 Lozenetz, Bulgaria, June 2008. Revised Selected Papers. Springer (2008)

19. Narahari, M., Akilu, S., Jaafar, A.: Free convection flow of a nanofluid past an isothermal inclined plate. Appl. Mech. Mater. 390, 129-133 (2013)

20. Narvaez, J.A., Veydt, A.R., Wilkens, R.J.: Evaluation of nanofluids as potential novel coolant for aircraft applications: the case of de-ionized water-based alumina nanofluids. ASME J Heat Transf. 136(051), 702 (2014)

21. Natesan, S., Jayakumar, J., Vigo-Aguiar, J.: Parameter uniform numerical method for singularly perturbed turning point problems exhibiting boundary layers. J. Comput. Appl. Math. 158, 121-134 (2003)

22. Pohlhausen, E.: Der warmeaustausch zwischen festen korpern und flussigkeiten mit kleiner reibung und kleiner warmeleitung. J. Appl. Math. Mech. 1, 115-121 (1921)

23. Sathiyamoorthy, M., Chamkha, A.: Effect of magnetic field on natural convection flow in a liquid gallium filled square cavity for linearly heated side wall(s). Int. J. Therm. Sci. 49, 1856-1865 (2010)

24. Shanthi, V., Ramanujam, N., Natesan, S.: Fitted mesh method for singularly perturbed reactionconvection-diffusion problems with boundary and interior layers. J. Appl. Math. Comput. 22, 49-65 (2006)

25. Siddiqa, S., Hossain, M., Saha, S.C.: The effect of thermal radiation on the natural convection boundary layer flow over a wavy horizontal surface. Int. J. Therm. Sci. 84, 143-150 (2014)

26. Tiwari, R.K., Das, M.K.: Heat transfer augmentation in a two-sided lid-driven differentially heated square cavity utilizing nanofluids. Int. J. Heat Mass Transf. 50, 2002-2018 (2007) 